\title{
Beaumarchais et ses musiciens : Paisiello, Rossini, Mozart
}

\section{Pierre Michot}

\section{(2) OpenEdition \\ 12 Journals}

Édition électronique

URL : http://journals.openedition.org/edl/986

DOI : $10.4000 /$ edl. 986

ISSN : 2296-5084

Éditeur

Université de Lausanne

\section{Édition imprimée}

Date de publication : 15 décembre 2016

Pagination : 17-32

ISBN : ISBN 978-2-940331-50-5

ISSN : 0014-2026

\section{Référence électronique}

Pierre Michot, « Beaumarchais et ses musiciens : Paisiello, Rossini, Mozart », Études de lettres [En ligne], 4 | 2016, mis en ligne le 15 décembre 2019, consulté le 15 décembre 2020. URL : http:// journals.openedition.org/edl/986; DOI : https://doi.org/10.4000/edl.986 


\section{BEAUMARCHAIS ET SES MUSICIENS, PAISIELLO, ROSSINI, MOZART ${ }^{1}$}

Nombreux sont les compositeurs qui ont mis en opéra Le barbier de Séville de Beaumarchais. Seuls Paisiello (1782) et plus encore Rossini (1816) ont survécu. Les comparer au gré de quelques moments choisis fera certes couronner la verve du second, mais rendra aussi justice à quelques belles trouvailles du premier. Quant à Mozart, il est le seul à s'être affronté au Mariage de Figaro (1786). Le personnage de Rosine prend des couleurs très contrastées, tendrement mélancolique chez Paisiello, mutine et déterminée chez Rossini, blessée et déchirante chez Mozart.

Pierre Augustin Caron de Beaumarchais fait représenter son Barbier de Séville en 1775. Chute à la première, mais vif succès par la suite et triomphe dans toute l'Europe, jusqu'à Saint-Pétersbourg. C'est là-bas que pour complaire à Catherine II qui avait apprécié la pièce française, le compositeur italien Giovanni Paisiello en fait un opéra, en 1782, sept ans après la création de la comédie de Beaumarchais. En 1784, c'est la suite du Barbier, Le mariage de Figaro, que Beaumarchais fait jouer à la Comédie française. Deux ans après seulement, alors que la pièce est encore interdite sur les scènes impériales autrichiennes, Da Ponte et Mozart donnent Le nozze di Figaro au théâtre de Vienne.

En 1816, alors que l'opéra de Paisiello est encore joué partout avec succès, Rossini ose reprendre le même sujet et donne son propre

I. A l'origine de cet article, une conférence de présentation du Barbier de Séville de Rossini pour l'Association genevoise des amis de l'opéra et du ballet, en 1983, et une introduction aux Noces de Figaro ("De Beaumarchais à Mozart») à l'Université de Lausanne (Grange de Dorigny), en 2013. 
Barbiere di Siviglia sur la scène du Teatro Argentina de Rome. Comme à la création de la pièce de Beaumarchais, chute à la première (une cabale orchestrée par les admirateurs de Paisiello n'y est sans doute pas étrangère), puis vif succès par la suite et triomphe rapide dans toute l'Europe, qui relègue bien vite dans l'oubli l'œuvre de Paisiello.

On oubliera les noms des douze autres compositeurs qui ont écrit un Barbier de Séville, de 1776 à 1924, où un certain Alberto Torazza (il était le troisième à avoir cette douce folie) a eu l'audace, sans lendemain, de reprendre le livret même de Rossini!

Paisiello et Rossini, confrontés sur le même scénario, c'est le sujet principal de cet article. Entre Le barbier et Le mariage, les personnages de Beaumarchais ont vieilli de trois ans. Mozart ne sera abordé que sous l'angle de la seule Rosine, devenue Comtesse Almaviva.

\section{Le barbier de Séville de Beaumarchais}

C'est l'histoire d'un oiseau en cage, qui chante et gazouille derrière ses barreaux, mais n'aspire qu'à s'envoler, dès le moment où un rossignol enamouré est venu lui donner le goût de la liberté. L'oiseau, c'est la jeune Rosine, orpheline de noble naissance; la cage, c'est la maison du docteur Bartholo, tuteur de la jeune fille; malgré son âge, il a décidé d'épouser sa pupille. Le rossignol en amour, c'est le Comte Almaviva, Grand d'Espagne, qui, incognito, sous le costume et le nom de l'étudiant Lindor, tente l'impossible pour approcher la jeune fille. Sous les fenêtres de Bartholo, il retrouve par hasard Figaro, qui fut autrefois son valet et qui a multiplié les métiers et les voyages avant de s'établir barbier à Séville. Heureuse rencontre, car Figaro a ses entrées dans la place: il rase le Docteur et sert de factotum à la maisonnée. Figaro suggère au Comte de se déguiser en soldat, muni d'un billet de logement, et de se faire héberger par Bartholo. Le stratagème ne mène pas loin, mais Lindor-Almaviva a pu approcher Rosine et lui remettre un billet doux. Nouveau travestissement, Almaviva se transforme en Don Alonzo, et prétend remplacer Bazile, maître de musique de Rosine, pour la leçon de chant de celle-ci. Son déguisement est aussi percé à jour, mais le Comte a eu le temps d'avertir Rosine qu'il viendra l'enlever dans la nuit. Mis au courant du rendez-vous, Bartholo fait enlever l'échelle qui a permis au Comte et à Figaro de s'introduire dans la maison. Ce qui coupe la 
retraite des amants, mais leur laisse le temps de profiter du notaire que le Docteur avait fait quérir pour son propre mariage. Le Comte Almaviva reconnu comme tel, a épousé Rosine, et Bartholo ne peut que déplorer son erreur, celle qui donne son sous-titre à la pièce: La précaution inutile.

L'historien du théâtre nous fera remarquer que le schéma de l'intrigue est déjà celui de L'école des femmes de Molière où un barbon veut épouser la pupille qu'il séquestre, mais doit finalement céder devant les droits d'un jeune amant. On repérera aussi tout ce qui ressortit à la tradition de la commedia dell'arte: les épisodes farcesques, les travestissements nombreux du jeune amoureux, en soldat ivre ou en maitre de musique, l'aubade sous la fenêtre de l'aimée, les nombreux billets qu'il faut transmettre, échanger, dissimuler, tout autant que l'échelle au balcon.

Le valet astucieux chargé d'aider aux entreprises de son maître remonte, à travers Molière, jusqu'à la comédie antique, mais la figure particulière de Figaro, ses avatars, ses voyages et ses revers de fortune, ses métiers accumulés et ses compétences diverses, tout cela fait penser au roman picaresque, mais aussi à la biographie aventureuse de Beaumarchais. Figaro, a-t-on dit, c'est l'homme nouveau, celui que la Révolution mettra en place. Sans doute, mais on dira du schéma de cette pièce qu'il est encore d'Ancien Régime: le valet est au service du maître pour duper le bourgeois, la Noblesse trompe le Tiers-Etat en s'appuyant sur le Peuple, qui met encore ses volontés d'émancipation et de liberté au service d'un grand. Figaro en fait son profit, mais dans la pièce suivante, son réveil sera dur: quand il voudra lui-même prendre femme, c'est le Comte qui se dressera contre lui. Son alliée, ce sera Rosine devenue Comtesse.

A tous ces éléments, dans cette pièce qui est à la convergence de multiples courants, s'ajoute encore l'influence du théâtre réaliste de Diderot $^{2}$ : la peinture des types sociaux, comme ce Bazile, organiste et intrigant, parasite toujours au service de plus riches et plus puissants que lui, toujours prêt à trahir pour un plus généreux. Car l'or mène l'intrigue: Figaro aiguise son astuce à l'odeur de ce métal, et Bazile trouve toujours qu'une bourse bien garnie est un argument sans réplique.

Enfin, brochant sur le tout et animant les dialogues, l'esprit brillant, l'ironie voltairienne, le scepticisme mordant, compensé par une gaieté

2. Voir les Entretiens sur "Le fils naturel" et De la poésie dramatique, in Euvres esthétiques. 
constante, et le goût de la formule frondeuse qui fait autant de mots d'auteur, du type:

Aux vertus qu'on exige dans un domestique, Votre Excellence connaîtelle beaucoup de maîtres qui fussent dignes d'être valets? ${ }^{3}$

Ce brillant du dialogue, c'est bien sûr ce qui se perdra dès qu'on transformera la comédie en livret d'opéra. Ce qui reste, c'est l'invention dans l'intrigue, la variété des situations, la netteté des personnages affrontés, toutes choses que le musicien pourra faire valoir au gré de son talent ou de son génie.

\section{Le barbier à l'opéra: Paisiello et Rossini}

Paisiello et son librettiste Petrosellini, Rossini et son librettiste Sterbini vont être confrontés au gré d'une courte scène d'action, la «Scène de la barbe», qui vient au terme d'un épisode dont il convient de rappeler le détail.

Almaviva s'est introduit dans la maison de Bartholo déguisé en Don Alonzo, élève de Basile et s'annonce comme remplaçant du maître de musique, qu'il prétend malade et forcé de garder le lit, pour donner sa leçon de chant à Rosine, en présence de Bartholo. Coup de théâtre: arrivée surprise de Bazile, étonné de voir Bartholo étonné; le Comte réussit à convaincre Bazile, bourse en main, qu'il est bien malade et qu'il lui faut rentrer chez lui.

Tous les personnages prennent congé - «Bonsoir Bazile», puis Alonzo et Rosine reprennent leur leçon de chant: le Comte en saisit le prétexte pour informer sa bien-aimée de l'enlèvement projeté. Pendant ce temps Figaro entreprend de faire la barbe au Docteur et fait tout ce qu'il peut pour l'empêcher de voir le couple et d'entendre ses propos. Peine perdue, Bartholo les surprend et entre en fureur.

On se référera à notre tableau en trois colonnes en écoutant la musique ${ }^{4}$. A gauche, le texte de la comédie, au centre Paisiello (Acte II, n ${ }^{\circ} 15$ de la partition). Son livret n'est qu'une traduction, réplique par réplique, du texte de Beaumarchais, simplement mis en vers, dans un

3. Le barbier de Séville, Acte I, scène 2 (Beaumarchais, Théâtre complet, p. 174).

4. Cf. Annexe. 
italien assez plat qui banalise le style du français et uniformise le rythme. L'incessante répétition des mêmes paroles contredit le mouvement général, mais cette jolie musique a de la vivacité, sans trop de surprise. Dans la première partie, elle avance en suivant consciencieusement le découpage des répliques, sans souci de caractériser les personnages: le chant est assez monotone sur une métrique trop régulière. Les violons ont un trait vif qui stimule la démarche, mais des accords sur des cadences scandent lourdement la fin des répliques. De même que Beaumarchais terminait sa scène par un "Il est fou" répété par tout le monde, Paisiello joue sur le mot pazzo pour faire culminer son ensemble. Ce qui frappe dans la structure du morceau, c'est que la colère de Bartholo n'introduit aucune rupture: quand il surprend le couple et entre en rage, la musique a continué dans le même mouvement et a repris un motif déjà utilisé. La césure est placée après, quand le Comte prend la défense de Rosine et qu'éclate la révolte de la jeune fille. D'où l'ensemble final qui réunit les voix, et oppose les personnages à trois contre un.

Dans Le barbier de Rossini (suivre la colonne de droite, acte II, $\mathrm{n}^{\circ} 13$ de la partition), le librettiste garde la structure globale de Beaumarchais, mais il concentre, il élague, il déplace les accents. L'écriture du texte n'est pas fidèle au modèle français, elle est tout entière pensée pour la musique à venir. Dans ce qui précède, le très célèbre Buona sera a donné lieu à un ensemble magistral, relancé par la fausse rentrée de Bazile que tout le monde croyait déjà sorti. Quand l'orchestre scande le via di qua ("sortez d'ici») qui l'expédie, une jolie trouvaille se prépare: une modulation relance l'intérêt, une cadence suspensive fait obligatoirement enchấner sur le quatuor. On fait tout de suite l'économie des commentaires sur la santé de Bazile et on passe à la scène de la barbe. Deux éléments sont nettement différenciés: le rasage et le dialogue furtif des amoureux. D'un côté Figaro dont la dextérité de barbier semble visualisée par un thème volubile des violons qui, jouant près du chevalet, ont une sonorité toute particulière. Ce thème est léger comme les elfes, avec de brusques détours soulignés par les bois. De l'autre côté la leçon continue: sous prétexte d'exercices vocaux, Alonzo et son élève placent les paroles confidentielles concernant l'enlèvement, sur une seule note, recto tono, avec une jolie arabesque des violons pour l'accompagner et un envol de vocalises à la fin. Délicieux moment de tendresse. Quand Bartholo les surprend, c'est un nouvel ensemble, un tourbillon de mots et de notes, les mots n'étant d'ailleurs choisis que parce qu'ils sont déjà en soi de la musique: 
Ma zitto Dottore, la testa vi gira ("Mais du calme, Docteur, la tête vous tourne»), ou: Bricconi, birbanti, su fuori furfanti ("Coquins, brigands, allons, dehors, canailles»). Ce mouvement frénétique, cette délicieuse mécanique cliquète et crépite, avec un orchestre aux sonorités acidulées: moulinets de violons, petite fanfare de clarinettes, avec des sistres et des piccolos qui font comme les grelots de l'attelage.

La césure était au milieu de la scène: Almaviva murmure sur un orchestre devenu presque muet, et Bartholo d'abord hilare, semble, en récitatif, jubiler de sa découverte. Sa rage éclate ensuite et met en marche le mouvement frénétique qui est du pur Rossini, mais semble annoncer avec cinquante ans d'avance les galops d'Offenbach.

Première leçon de cette comparaison: indépendamment de la qualité intrinsèque des deux musiques, la liberté prise avec le modèle est plus payante que la servilité. Chez Rossini, tout devait culminer dans ce pur moment musical, où le mouvement n'est plus dans les dialogues et le jeu, mais dans les voix et l'orchestre. Et pour cela on a fait le sacrifice, gaiement consenti, des répliques importantes où Rosine s'insurge contre son esclavage et donne sa foi à celui qui l'en arrachera.

$\mathrm{Si}$ - au-delà de cette simple scène - on considère l'ensemble des deux opéras, on vérifie ce que nous avons constaté ici: Paisiello suit la construction de Beaumarchais, le découpe en récitatifs et en airs et ensembles, mais ne crée pas de vraie structure musico-théâtrale. Rossini et Sterbini en prennent à leur aise, créent un nouveau découpage, et construisent deux actes qui répondent aux lois de l'opéra buffa telles que Mozart a puissamment contribué à les établir. L'introduction du chœur impose une nouvelle scène dès le début du premier acte: celle de l'aubade du Comte accompagné de musiciens. Si le chœur réapparaît à la fin de l'acte, c'est pour permettre un grand Finale. Donc le livret invente un nouvel épisode: le tapage qu'on fait chez Bartholo attire la Garde, conduite par un officier prêt à arrêter Almaviva le faux soldat.

Ainsi le mouvement propre au théâtre musical, qui est structuration de la durée, économie des tensions et des détentes, progression par la convergence des effets, recrée par ses moyens à lui ce qui chez Beaumarchais naît du brillant des dialogues et du rebond des situations. L'excessive fidélité à la lettre, chez Paisiello, finit par détruire l'essence de Beaumarchais; la fidélité à l'esprit, chez Rossini, reconquiert cette essence par les moyens qui sont ceux de l'opéra buffa. 
Ne soyons pourtant trop injuste pour Paisiello qu'il ne faut pas juger sur cette seule scène. La comparaison ne devrait pas aboutir à distribuer des prix, mais à caractériser des styles.

Dans une autre scène (acte II, scène $7, n^{\circ} 7$ ), Paisiello est sans concurrence, tout simplement parce que cette scène met en jeu des personnages secondaires que Rossini a supprimés. C'est le passage amusant où Bartholo essaie d'interroger ses deux serviteurs, l'Eveillé (lo Svegliato) et la Jeunesse (il Giovinetto), pour savoir s'il est entré quelqu'un chez Rosine. Mais Figaro a fait des ravages pour neutraliser les Argus: puisant dans sa pharmacie, il a donné un narcotique à l'Eveillé et un sternutatoire à la Jeunesse. Cela donne un ensemble où les violons baillent aussi fort que l'Eveillé, et où la Jeunesse éternue consciencieusement en mesure. La progression du morceau, d'abord Duo, ensuite Trio, joue sur l'effet d'accumulation des bâillements auxquels viennent s'ajouter les éternuements.

Rossini a sans doute jugé que, là, Paisiello était insurpassable. Il n’a pas cherché à l'affronter sur le même terrain. Mais à bien d'autres endroits, on a le sentiment qu'il n'a pas hésité à prouver qu'il était capable de faire mieux, en exploitant parfois des trouvailles qui reviennent à Paisiello. C'est le cas par exemple au début du $2^{\mathrm{e}}$ acte: l'invention de Paisiello est réelle, Rossini la reprend et la dépasse, car il y introduit la variété et cette vivacité qui est au principe de son génie. Il s'agit de l'entrée d'Almaviva déguisé en Don Alonzo: le personnage que joue ici le Comte est aussi cérémonieux et poli qu'était bruyant et brutal son soudard du $1^{\text {er }}$ acte. Beaumarchais commençait sa scène par cette salutation d'Alonzo: "Que la paix et la joie habitent toujours céans!», puis il enchaîne. C’est cette unique réplique qui donne lieu à tout le duo $\left(n^{\circ} 12\right)$. Tout l'effet comique tient à la répétition, ad nauseam, de la formule: Pace e gioia sia con voi ("Paix et joie soient avec vous»), sur un ton traînant, psalmodiée toujours sur la même note, avec des violons doucereux sur des basses dodelinantes. Belle supériorité du musicien sur l'auteur dramatique: Beaumarchais n'avait pas pensé à un tel effet théâtral. C'est très réussi. On se dit pourtant que l'effet ne se renouvelle guère.

Rossini reprend presque textuellement l'idée de Paisiello ( $\left.{ }^{\circ} 10\right)$. Mais il l'améliore tellement qu'on ne peut que le féliciter de ce plagiat. Chez Paisiello, Alonzo répétait inlassablement ses salutations et Bartholo s'énervait tout de suite: Ohimè che noia ("quel ennui!»), qui rime avec gioia («joie»). On ne sentait pas la naissance de l'irritation et on faisait 
du sur-place. Rossini nous montre Bartholo qui commence par répondre très poliment, par remercier: Mille grazie, obbligato in verità ("Mille mercis, je suis votre obligé»); qui dit ensuite: "c'est bon, j'ai compris», basta basta, ho capito, et qui finit par s'agacer et crier. Quant au thème qu'invente Rossini, avec ses trilles et ses appoggiatures, il suggère, en plus de la psalmodie confite en dévotion, un dos propre à se courber pour la révérence, et finalement une hypocrisie digne d'un élève de Bazile. Et surtout, Rossini introduit un élément de renouvellement du duo par un effet de contraste: les salamalecs alternent avec des apartés, sur des cascades de triples croches, où Bartholo se demande s'il n'a pas déjà vu cette tête-là quelque part et où le Comte est anxieux de savoir comment passe son nouveau déguisement. A la reprise, comme le Comte se dit que décidément le vieux ne le reconnaît pas et qu'il va pouvoir parler à son aimée, sa phrase se fait chantante et son lyrisme s'envole au-dessus du débit rapide de l'autre voix. C'est alors l'euphorie: le pur plaisir du comique avec en plus une délicate touche de poésie. Il y a là une subtilité que Mozart n'aurait peut-être pas désavouée.

A partir de ces extraits, mais en réécoutant l'ensemble de l'opéra, on pourrait établir une sorte de vocabulaire musical rossinien. Par exemple le parlato rapide typique de la basse bouffe mozartienne (pensez à Leporello!), le texte débité à toute allure, devient un trait caractéristique de l'écriture vocale de Rossini, jusqu'à atteindre à la plus folle virtuosité. Il y a bien sûr aussi le fameux crescendo, il y a le rythme ostinato et le trait mélodique récurrent qui structure tout un morceau, le jeu sur la langue, consonnes ou onomatopées, et surtout la virtuosité des vocalises, toute cette technique de l'agilità qui nous fait nous souvenir d'une chose importante.

$\mathrm{Au} \mathrm{XVIII}$ e siècle, on sépare très nettement, dans l'opéra italien, le genre opera buffa (sujets empruntés au quotidien, personnages familiers, formes simples et écriture vocale sans grande exigence virtuose) et le genre opera seria (sujets mythologiques ou historiques, personnages drapés dans le haut style, écriture vocale d'une extrême virtuosité, privilège des grands castrats et des grandes prima donna, le bel canto au sens propre du terme, c'est-à-dire le chant orné, la volubilité, la broderie rapide et légère, la maîtrise de la couleur). La caractéristique de Rossini, qui hérite de cette époque et la prolonge, c'est de faire entrer dans l'opéra buffa les exigences de ce bel canto-là. 
Dans l'écriture vocale, le poids de cette tradition, autant que le recours aux trucs propres à Rossini, détermine un style musical où l'invention joue à partir de et sur ces mille formules. La liberté créatrice et le procédé mille fois repris ne sont pas antagonistes. Ce style permet de traiter à merveille la mécanique de l'intrigue, de styliser les situations, de faire pétiller la musique. Mais ce style a-t-il assez de souplesse pour permettre une véritable caractérisation psychologique des personnages?

\section{Le personnage de Rosine}

C'est ce qu'il nous faut voir encore, en comparant cette fois non pas une scène mais un personnage. Ajoutons Mozart à nos deux Italiens, et voyons comment, chez les trois musiciens, se présente la figure de Rosine. Brisons la chronologie et commençons par Rossini.

Le premier air de Rosine ( $\mathrm{n}^{\circ}$ 5), Una voce poco fa ("Une voix à l'instant a résonné dans mon cœur»), la désigne, et la dessine à merveille, car elle prend soin de faire son propre portrait. Un portrait à deux faces: soumise, et combative. Io sono docile, chante-t-elle d'abord. Je suis docile, respectueuse, obéissante, douce, amoureuse, je me laisse conduire. "Mais», ma - et c'est le plus célèbre ma de tout le répertoire Ma se mi toccano, si on me touche là où est mon point faible, je serai une vipère, una vipera sarò. Double aspect de Rosine que Maria Callas faisait entendre de manière inoubliable. Docile, mais aussi rebelle: Cento trappole prima di cedere farò giocar ("cent pièges qu'avant de céder, je saurai faire jouer»). La même détermination conduisait la première partie de l'air:

La voix de Lindor a touché mon cœur, c'est décidé, Lindor sera à moi, je vaincrai; le tuteur fera obstacle, j'aiguiserai ma ruse, il cédera et je vivrai heureuse ${ }^{5}$.

Voilà cette Rosine rossinienne: décidée à se battre, prête à tout pour obtenir ce qu'elle a choisi de vouloir. Une petite bonne femme tout ce qu'il y a de plus impatient et déterminé. Charmante, adorable, certes, parce qu'assez souple pour savoir enrober de miel ses exigences. Surtout pas une virago, parce que très jeune fille, avec sa délicatesse et sa

5. C'est nous qui traduisons. 
fraîcheur. Mais une toute maline, une rusée qui en remontre à Figaro lui-même.

Dans sa Vie de Rossini, Stendhal fait cette remarque:

La cavatine de Rosine Una voce poco fa est piquante; elle est vive, mais elle triomphe trop. Il y a beaucoup d'assurance dans le chant de cette jeune pupille persécutée, et bien peu d'amour ${ }^{6}$.

Bien peu d'amour en effet, comme si la tête était trop froide pour que le cœur s'attendrisse. Quelques instants plus tard, quand Figaro lui confirme que c'est bien elle qu'aime le garçon qu'elle a vu sous sa fenêtre, elle réagit avec émotion certes (Dunque io son, c'est donc moi, tu ne me trompes pas, l'heureuse élue). Mais son aparté, chargé de vocalises, confirme qu'elle garde la tête froide: Già me l'ero immaginata ("Je me l'étais bien imaginé, et je le savais avant toi»).

Commentaire de Stendhal:

Je ne croirai jamais que l'amour chez une jeune fille, même à Rome, soit à ce point privé de mélancolie, et j'oserai dire d'une certaine fleur de délicatesse et de timidité 7 .

Privé de mélancolie: en effet, l'amour selon Stendhal doit s'ombrer de davantage de tendre rêverie. Ici, Rosine s'émerveille d'apprendre qu'elle est aimée, mais avec des accents où le triomphe a plus de part que l'émotion. Ce qu'il y a pourtant de merveilleux, c'est que la courbe musicale, la vivacité de la vocalisation adoucit ce que ce caractère pourrait avoir d'un peu dur: la musique lui donne une désinvolture rieuse et un enjouement gracieux.

La Rosine de Paisiello est totalement différente, et bien plus proche de celle de Beaumarchais, qui a beaucoup de délicatesse pudique et une vraie générosité amoureuse. Autant Rossini nous montre une fille rusée, malicieuse et énergique, autant Paisiello la voit résignée et plaintive. Dans sa cavatine Giusto ciel ( $\left.{ }^{\circ} 11\right)$, au-delà de la convention mélodique, il y a cet accompagnement élégiaque de la clarinette et du basson, il y a ces accents de délicatesse touchante, avec ces surprises à la fin des phrases, qui font de cette Rosine de Paisiello une fleur fragile, une créature inquiète et tendre, qui a bien de la peine à s'insurger contre son

6. Stendhal, Vie de Rossini, p. 221.

7. Ibid., p. 223. 
tyran et qui ne peut que supplier le ciel de donner à son cour la paix qu'il ne connaît pas.

Autre passage encore plus touchant de la Rosine de Paisiello, l'air de la leçon de chant $\left(\mathrm{n}^{\circ} 13\right)$ : au milieu d'une évocation très "Petit Trianon" d'une bergère avec ses moutons, elle dit soudain ceci : Io piango afflitta e sola ("Je pleure affligée et seule, pauvre pastourelle, non mon agnelle perdue, mais le berger Lindor $»)$. Musique toute simple, et d'une expression infiniment touchante. Ces courbes mélodiques qui sont comme des soupirs, ces fins de phrases qui sont comme des coups au cœur, et ces silences désemparés, tout cela rappelle ce que Stendhal disait de Paisiello:

Ses émotions ne s'élèvent guère au-delà de la grâce, mais il a excellé dans ce genre; sa grâce est celle du Corrège, tendre, rarement piquante, mais séduisante, mais irrésistible ${ }^{8}$.

On n'a pas cité l'indication scénique qui accompagne ce passage de la leçon de chant:

Bartholo s'endort en écoutant cet air. Le comte (qui est censé l'accompagner au clavecin) se hasarde à prendre une main de Rosine et à la baiser. L'émotion ralentit la voix de Rosine, qui faiblit et finit par manquer au milieu de la cadence 9 .

Cette Rosine qui pleure son Lindor perdu, comment ne pas y voir, y entendre une préfiguration de la Rosine de quelques années plus tard, la Comtesse délaissée du Mariage de Figaro. Car on tombera d'accord: il est bien difficile d'imaginer que les personnages du Barbier tels que Rossini les a fait vivre par sa musique, même muris de quelques années, deviennent ceux des Noces de Figaro. Impossible de penser que la Rosine de Rossini, si décidée, "plus rusée qu’amoureuse», devienne la Comtesse de Mozart, si vulnérable, si sensible. En revanche, il n'est pas impossible de voir dans la Rosine de Paisiello des traits qui s'épanouiront dans la Comtesse de Mozart. Chez Paisiello, ce n'est qu'une esquisse, sans doute, mais dans la même cohérence psychologique.

En les faisant passer du Barbier au Mariage, Beaumarchais avait beaucoup transformé ses personnages: tous ont mûri, ils ont vieilli. C'est vrai de Figaro, du Comte, et plus encore de la Comtesse. Mozart l'a

8. Stendhal, Vie de Rossini, p. 32.

9. Beaumarchais, Théâtre complet, p. 209. 
bien compris. Rosine devenue Comtesse Almaviva s'exprime avec une profondeur nouvelle dans son air le plus connu, et le plus beau, le Porgi amor qui ouvre le $2^{\mathrm{e}}$ acte $\left(\mathrm{n}^{\circ} 21\right)$. On y retrouvera la jeune fille, devenue femme, on y retrouvera sa grâce délicate, sa féminité pudique, mais on y découvrira une sensualité épanouie, dont la maturité de la voix est le signe; on y admirera une noblesse affirmée. On y reconnaît la blessure qu'esquissait la petite Rosine, mais ici cette blessure est béante, c'est la nudité du cœur délaissé, c'est l'appel à la mort si doit s'éteindre la flamme de l'amour, c'est mille choses en trois minutes, bref, c'est Mozart.

\section{Conclusion: chacun à sa place}

Plus d'une douzaine de compositeurs ont mis en musique Le barbier de Séville de Beaumarchais, certains n'hésitant pas à venir après des chefsd'œuvre confirmés. Pour Le mariage de Figaro, il n'y en a eu qu'un. Rossini n'a pas hésité à refaire le travail de Paisiello. Jamais il n'aurait osé refaire Mozart. Si bien qu'on se dit que les choses se sont fort bien combinées et que Beaumarchais a eu bien de la chance.

Le barbier de Séville, une action bien dessinée, des situations d'un comique déclaré, des travestissements farcesques, des caractères bien typés, tout cela appelait Rossini, sa mécanique impeccable, sa gaieté sans mélange, son brillant euphorique, sa netteté simple et efficace.

Le mariage de Figaro, une action compliquée, des désirs entrecroisés, des jalousies aiguës, la nostalgie du bonheur que l'on a perdu ou qu'on vous refuse, des personnages subtils, avec leurs revendications et leurs désillusions, tout cela, c'était le monde de Mozart et de lui seul, avec son sens de l'ambiguïté, sa force et sa tendresse, son sourire qui s'embue de larmes.

Pour le plaisir d'une spéculation un peu absurde, essayons de renverser les rôles. Imaginons un instant Rossini compositeur du Mariage de Figaro: il aurait magistralement donné son rythme à la Folle Journée, stylisant les ensembles, traitant l'imbroglio dans des formes simples, avec une gaieté débridée, faisant vocaliser tout le monde dans un spumante allègre et ensoleillé. Mais la douleur de la Comtesse, le désir du Comte, le frémissement de Chérubin, la finesse de Suzanne, l'amertume de Figaro? Presque impensable. 
Quant à imaginer Mozart composant Le barbier de Séville? Il n'est pas interdit de rêver. Des ensembles foisonnants de détails auxquels on n'a jamais pensé, un orchestre délicat et un léger voile de brume sur le soleil de Séville, un Comte sans doute plus grand seigneur que Rossini ne l'a voulu, une Rosine piquante, mais capable, elle, de mélancolie stendhalienne, un Bartholo qui aurait pu émouvoir. Et peut-être, comme dans Così fan tutte, un immense abîme révélé par la musique sous les pieds des amants...

Il n'est pas interdit de rêver à ce qui n'existera jamais. Mais la réalité reste miraculeuse: les deux comédies de Beaumarchais ont exactement trouvé les musiciens qui leur convenaient. On s'amuse follement au Barbier de Rossini, et on rêve, on vibre, on pleure aux Noces de Mozart. En vérité, l'histoire de la musique est fort bien faite.

Pierre Мiснот

Professeur honoraire d'histoire de la musique au Conservatoire de Genève 


\section{BIBLIOGRAPHIE}

Beaumarchais, Jean-Pierre de, Théâtre complet, Paris, Bibliothèque de la Pléiade, 1957.

Colas, Damien, Rossini, l'opéra de lumière, Paris, Gallimard, 1992.

Diderot, Denis, Euvres esthétiques, Paris, Garnier, 1965.

Mancini, Roland, Rossini, Paris, Fayard, 1993.

Мiснот, Pierre, Mozart, opéras, mode d'emploi, Paris, Ed. Premières Loges, 2006 (L'Avant-Scène Opéra).

Mozart, Wolfgang Amadeus, Les noces de Figaro. Paris, Ed. Premières Loges, 2007 (L'Avant-Scène Opéra, 135-136).

Rossini, Gioachino, Le barbier de Séville, Paris, Ed. Premières Loges, 2005 (L'Avant-Scène Opéra, 137).

Stendhal, Vie de Rossini, Lausanne, Editions Rencontre, 1960.

Stricker, Rémy, Mozart et ses opéras, fiction et vérité, Paris, Gallimard, 1980. 
ANNEXE
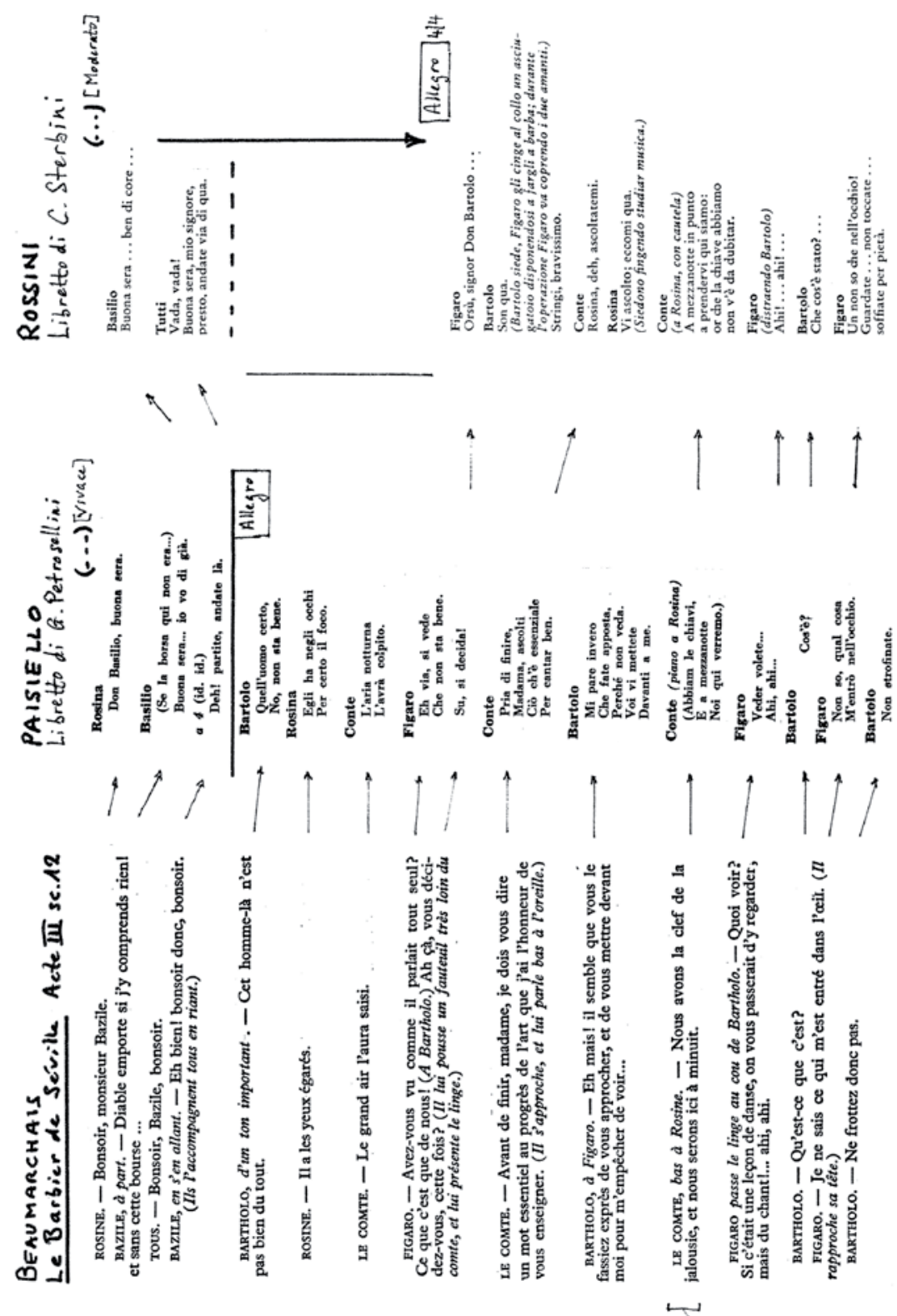
